\title{
Minuto Lumière no ensino de biologia evolutiva
}

Minuto Lumière in teaching

Minuto Lumière en la enseña evolutiva

Luís Gustavo da Conceição Galego

1 Professor Associado do Departamento de Ciênci Federal do Triângulo Mineiro (UFTM). Mestre, Doutor pela Universidade Estadual Paulista "Júlio de Mesquita Educação e Tecnologias pela Universidade Federal de Sãc Letras e Ciências Biológicas pela UNESP e em Pedagog http://lattes.cnpq.br/9101105344317706 - ORCID 6081-2700 - E-mail: luis.galego@uftm.edu.br 


\section{RESUMO}

O presente artigo é um relato de experiência sobre as potencialidades do Minuto Lumière, um recurso audiovisual inspirado nas produções cinematográficas geminais dos Irmãos Lumière, como instrumento de avaliação da disciplina "Evolução" de um curso de Licenciatura em Ciências Biológicas, cuja a oferta ocorreu remotamente em virtude da pandemia de COVID-19. Os alunos da disciplina, após orientações técnico-conceituais sobre o dispositivo, produziram e socializaram seus Minuto Lumière sobre a Evolução Humana. Essas produções constituíram uma das avaliações da disciplina e apresentaram a visão daqueles conhecimentos científicos a partir da perspectiva dos alunos, constituindo uma instrumento viável e multinível para aprendizagens em biologia evolutiva.

\section{PALAVRAS-CHAVE}

Ensino Remoto; Evolução Humana; Linguagem Audiovisual.

\section{ABSTRACT}

This article is an experience report on the potentialities of the Minuto Lumière, an audiovisual resource inspired by the Lumière Brothers' geminal cinematographic productions, as an instrument of evaluation of the discipline "Evolution" of a Bachelor of Science course in Biological Sciences, which the offer took place remotely because of the COVID-19 pandemic. The students of the discipline, after technical-conceptual guidance on the device, produced and socialized their Minuto Lumière with the theme of Human Evolution, which was one of the evaluations of the discipline and presented the vision of that scientific knowledge from the perspective of students, constituting a viable and multilevel for learning in evolutionary biology.

\section{KEY WORDS}

Remote Teaching; Human Evolution; Audiovisual Language.

\section{RESUMEN}

El presente artículo es un relato de experiencia sobre las potencialidades del Minuto Lumière, un recurso audiovisual inspirado en las producciones cinematográficas geminales de los Hermanos Lumière, como instrumento de evaluación de la disciplina "Evolución", de un curso de Licenciatura en Ciencias Biológicas, cuya oferta se produjo de forma remota debido a la pandemia de COVID-19. Los alumnos de la disciplina, después de orientaciones técnico-conceptuales sobre el dispositivo, produjeron y socializaron sus Minuto Lumière con el tema de Evolución Humana, que constituyó una de las evaluaciones de la disciplina y presentó la visión de aquellos conocimientos científicos desde la perspectiva de los alumnos, constituyendo un instrumento viable y multinivel para aprendizajes en biología evolutiva.

\section{PALABRAS CLAVE}

Enseñanza Remota; Evolución Humana; Lenguaje Audiovisual. 


\section{Introdução}

O ano de 2020 foi marcado por uma das maiores pandemias da história: a COVID-19. Essa pandemia promoveu um conjunto de ações que modificaram a dinâmica da sociedade, tais como o isolamento social e consequente interrupção das aulas das instituições de Ensino Superior e de Ensino Básico em todo o país.

A promoção de videoconferências passaram a constituir, por um lado, possibilidades para a integração entre professores e alunose, por outro, tem promovido diversas discussões, tais como a questão da acessibilidade dos alunos à internet ou mesmo das dificuldades do uso das tecnologias da informação e comunicação para abordagens diversas que possam envolver, por exemplo, assuntos relacionados ao uso das Tecnologias Digitais de Informação e Comunicação (TDIC) nas universidades.

Um recurso que utiliza TDIC e que se apresenta como um potencial instrumento avaliativo é o Minuto Lumiére, um dispositivo audiovisual sem edição e em plano sequência com duração de um minuto, idealizado a partir das experimentações geminais dos irmão Lumière e conforme proposição de Alain Bergala e Nathalie Bourgeois (BERGALA, 2008).

O presente trabalho apresenta um relato de experiência sobre as potencialidades do Minuto Lumière enquanto prática de avaliação na disciplina "Evolução" do curso de Licenciatura em Ciências Biológicas da Universidade Federal do Triângulo Mineiro (UFTM) e sua aplicabilidade em contextos de ensino remoto, fruto do isolamento social provocado pela pandemia de COVID-19 que assolou o mundo a partir do ano 2020.

\section{TDIC e o ensino remoto}

As TDIC podem contribuir para mudanças na abordagem do ensinar-aprender, e possibilitam a autonomia e autoria nos processos de aprendizagem (LINHARES et al., 2017). Silva (2006) ressalta ainda que o professor deve compreender as possibilidades multinível que as TDIC oferecem, permitindo a utilização simultânea de recursos escritos, imagéticos e sonoros, estimulando aprendizagens múltiplas, além de atender os diferentes tipos de aprendizagem (GUATTARI; ROLNIK, 2011).

A importância das TDIC em processos de aprendizagem é ressaltada por Linhares et al. (2017, p.28):

As reflexões que consideram o lugar das TIC na sociedade atual, pensadas sob a perspectiva da sociedade da informação e do conhecimento, são unânimes em reconhecer que as tecnologias digitais da comunicação exercem papel relevante na realização de atividades da vida cotidiana e daquelas direta ou indiretamente relacionas aos processos de aprendizagem. Nesse contexto, são exigidas mudanças nas abordagens pedagógicas, que deixam de reproduzir modelos de repetição para privilegiar práticas de aprendizagem 
voltadas para a formação de sujeitos autônomos, inovadores e que dominam as competências necessárias para o uso das tecnologias.

As TDIC permitem, portanto, uma ressignificação da educação, do papel do professor, da aprendizagem e da avaliação, além de aproximar a realidade dos estudantes, impregnada de tecnologias, com a da escola (McLUHAN, 2004). Além disso, as TDIC também permitem superar as barreiras espaço-tempo, uma vez que podem ser acessadas a qualquer tempo e, virtualmente, em qualquer lugar.

A pandemia de COVID-19 promoveu uma adaptação radical das TDIC pelo ambiente escolar, apesar da sua incorporação paulatina, nas práticas pedagógicas, que vinha ocorrendo ao longo dos últimos anos (CANI et al., 2020). O ensino remoto emergencial foi implantado em diversos sistemas educacionais do Brasil, levando professores e alunos a utilizar intensamente as TDIC na promoção do processo de ensino-aprendizagem, impondo a professores e alunos a incorporação de novos valores, prioridades, saberes e espaços de aprendizagem (CANI et al., 2020), além de uma superação de práticas pedagógicas mais tradicionais (RONDINI et al., 2020). Nesse sentido, a avaliação no Ensino Remoto deve, portanto, superar os aspectos classificatórios que muitas ferramentas presentes nos AVA tendem a enfatizar, buscando uma dimensão mais processual e permitindo, assim, uma maior percepção das aprendizagens, tanto por parte dos alunos quanto dos professores (ELIAS et al., 2015).

Uma alternativa à avaliação tradicional no contexto do ensino remoto é a utilização de estratégias que incorporem produções audiovisuais no processo, de forma a se potencializar as possibilidades que os meios digitais oferecem (LÉVY, 1993; MEDRADO; DINIZ. 2020). Uma dessas produções é a realização de curtas-metragens sem edição, com um minuto de duração, os chamados Minuto Lumière (BERGALA, 2008).

\section{O Minuto Lumière e a Biologia Evolutiva}

Minuto Lumière constitui um dispositivo audiovisual, inspirado nos filmes produzidos pelos Lumière no início do cinema, no final do séc XIX, que foi proposto por Alain Bergala e Nathalie Bourgeois (BERGALA, 2008). Essa abordagem audiovisual consiste na produção de um filme com duração de um minuto, que não requer uma técnica apurada, uma vez que a gravação é efetuada com a câmera parada, sem cortes ou edições, o que permite sua realização por meio de equipamentos de baixo custo. Isso torna a metodologia acessível para uma parcela significativa dos estudantes e, portanto, mais democrática (FRESQUET, 2010).

A proposta avaliativa, utilizando o Minuto Lumière, iniciou-se pela elucidação técnico-conceitual do dispositivo, que consiste em filmar um plano fixo horizontal em sequência (sem cortes), com duração de um minuto. Essa filmagem não deve 
ser editada e sonorizada, e é realizada preferencialmente em preto-e-branco. Essa metodologia de produção audiovisual recupera a infância do cinema e suas primeiras experimentações com as imagens em movimento, consistindo em uma experiência autoral, uma vez que o sujeito elege, dispõe e captura os elementos cinematográficos segundo suas escolhas políticas, culturais e estéticas (BERGALA, 2008).

O Minuto Lumière foi uma das avaliações da disciplina "Evolução", do curso de Licenciatura em Ciências Biológicas da Universidade Federal do Triângulo Mineiro (UFTM). Os alunos poderiam escolher entre os temas "Macroevolução" ou "Evolução Humana" e, a partir deles, produziriam individualmente um recurso audiovisual com a estrutura Minuto Lumière. Ressalta-se que, em virtude do cenário pandêmico pela COVID-19, a disciplina foi ministrada integralmente à distância, o que incluiu a realização das avaliações.

Os alunos foram orientados, por meio de uma vídeo-aula sobre Minuto Lumière, produzida e veiculada pelo canal 7Cine (https://www.youtube.com/ channel/UCZnSfBU305SEQ049DHr6900), disponível em https://www.youtube.com/ watch? $\mathrm{v}=\mathrm{JHZ3Sa0}-\mathrm{n} 54$ (acesso em 07/02/2021). Além disso, orientações adicionais ocorreram via Google Meet e a partir do Ambiente Virtual de Aprendizagem (AVA) da disciplina, disponibilizado na Plataforma Moodle. No AVA-Moodle foram disponibilizados alguns exemplos de recursos audiovisuais estruturados no formato Minuto Lumière: https://www.youtube.com/watch?v=-iGWCURYdF0, https://www.youtube.com/watch?v=2ua4WLh1kzs e https://www.youtube.com/ watch?v=fQyPvTYhwo0, todos com acesso em 07/02/2021. Ressalta-se que, em virtude da estrutura narrativa do Minuto Lumière, o planejamento é um momento fundamental para a construção do recurso, uma vez que não se pode contar com recursos de edição pós-filmagem, e a dicotomia visível/não visível no enquadramento deve ser metodicamente considerada na geração do sentido que se intenciona (COMOLLI, 2016).

Os alunos produziram 12 recursos audiovisuais do tipo Minuto Lumière, dos quais oito enfocaram a evolução do homem (Figura 1). Em virtude do predomínio de vídeos com essa temática, aqui optou-se por se analisar somente essas produções e desconsiderar as outras quatro sobre macroevolução. Um enfocou a agricultura, outro o bipedalismo, três deles a produção de ferramentas e os demais a importância do polegar opositor para movimentos acurados. 


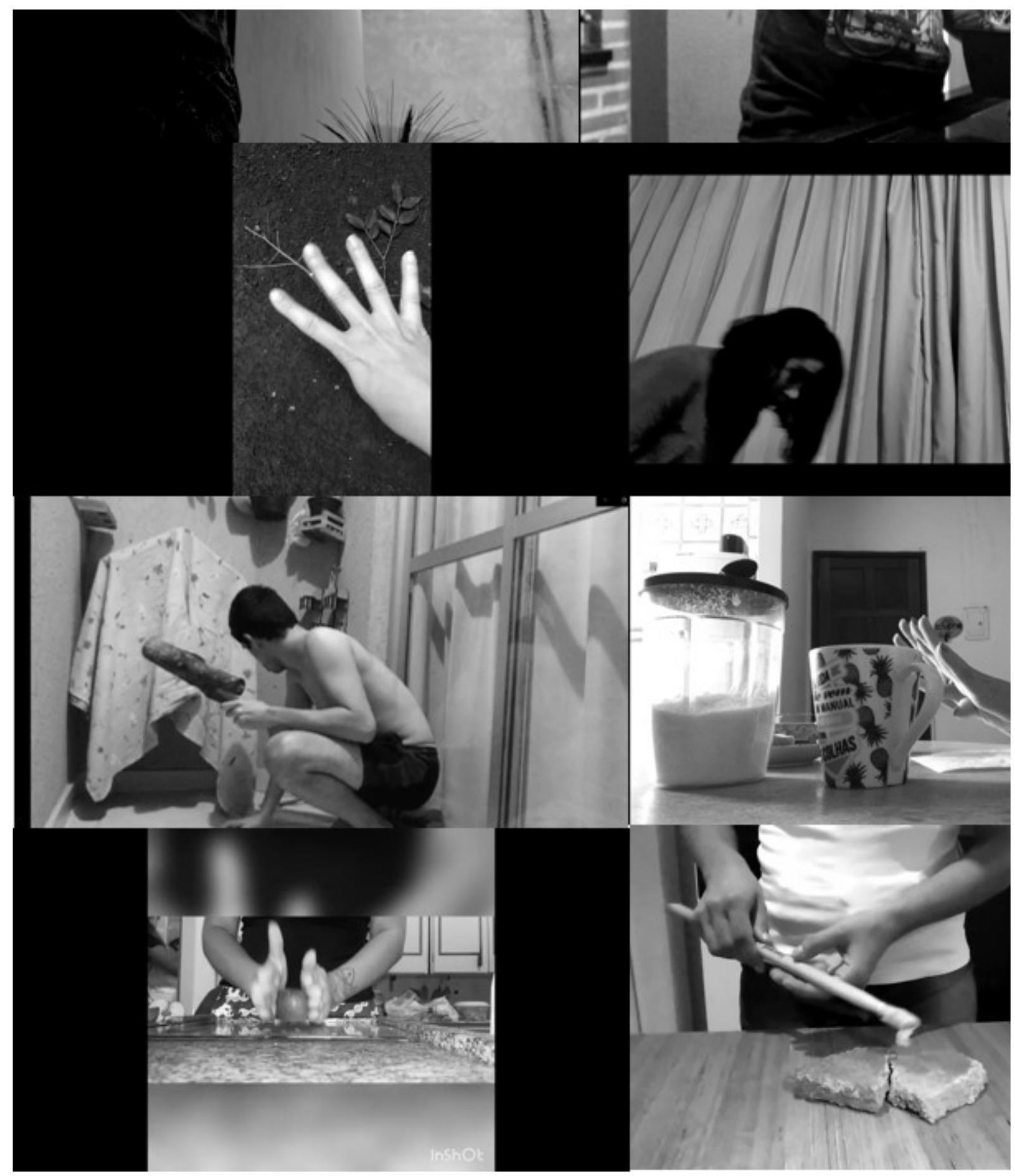

Fig 1.Screenshots dos Minuto Lumière produzidos por alunos de Licenciatura em Ciências Biológicas, sobre evolução humana.

As abordagens sobre evolução humana apresentadas nos vídeos constituem aquisições importantes dentro do processo biológico que culminou na humanidade: o bipedalismo, que proporcionou a exploração da paisagem geográfica; e o polegar opositor, relacionado a movimentos acurados que potencializam os processos criativos e manuais, como a construção de ferramentas e a domesticação das plantas (FUTUYMA; KIRKPATRICK, 2017). A escolha do enquadramento enfatizou os planos fechados (detalhe, médio e conjunto), o que é compatível com a intenção de se atribuir 
cientificidade à produção audiovisual, uma vez que esses planos são comumente utilizados em telejornais e documentários, o que gera, no inconsciente popular, uma dimensão de seriedade e de "verdade" científica (GALEGO; PEREIRA, 2020).

A ênfase em aspectos da evolução humana que são perceptíveis em seu próprio corpo, conforme ocorreu nas produções audiovisuais dos alunos, indica uma interação do conteúdo científico, discutido em uma atividade síncrona do ensino remoto, com a realidade dos estudantes. De fato, para ilustrar a importância do polegar opositor, os alunos filmaram suas mãos de forma a se reconhecer como sujeito da evolução. Em outras palavras, perceber-se enquanto elemento constituinte desse importante processo natural. Essa auto percepção também está presente nos Minuto Lumière com temáticas sobre bipedalismo, domesticação de plantas e construção de ferramentas: em todos eles, os alunos são sujeitos do conteúdo científico que estudaram.

O Minuto Lumière é uma das abordagens possíveis para a utilização do cinema em sala de aula. Considerando ainda a dimensão da produção audiovisual e suas potencialidades no ensino remoto, é possível ainda a proposição de outras avaliações que utilizem essa perspectiva do cinema na educação, tais como aquelas nas quais os alunos podem trabalhar em grupo, cada um desempenhando uma função (roteirista, diretor, ator, editor, etc.), a partir da organização desses grupos por meio de reuniões utilizando plataformas de webconferências tais como o Google Meet, e decisões sobre o roteiro, direção e edição tomadas coletivamente por meio de TDIC tipo whats app.

O cinema em sala de aula, nessa perspectiva, supera seu papel tradicional de ilustrador de conteúdos, e propostas que incluam a produção do audiovisual possibilitam novas dimensões para a aprendizagem (ALBUQUERQUE et al., 2017). Nesse sentido, as produções audiovisuais no estilo Minuto Lumière possibilitam uma estratégia avaliativa multinível que pode ser utilizada em contextos tanto presenciais quanto virtuais de ensino. $O$ cinema no contexto da formação universitária possibilita uma quebra no espaço e no tempo, potencializando as experiências criativas e estéticas (de DEUS; OLIVEIRA, 2018), além de oferecer outras possibilidades de avaliação de aprendizagens que considerem, além de conteúdos conceituais, outros saberes como o "fazer", o "sentir" e o "apreciar".

\section{Considerações finais}

O isolamento social produzido pela pandemia de COVID-19 produziu um impacto significativo na prática docente, uma vez que o ensino remoto emergencial surgiu como uma realidade nas sala de aula, tornando as TDIC imprescindíveis.

O processo de ensino-aprendizagem e a avaliação precisaram ser repensadas e novas possibilidades mediadas pelas TDIC foram incorporadas à ação do professor. Nesse sentido, o Minuto Lumière constitui uma abordagem audiovisual democrática, uma vez que não requer equipamentos, técnicas e edições mirabolantes e inacessíveis, 
sendo seu principal foco no planejamento e na percepção do visível/não visível do enquadramento.

A experiência com o Minuto Lumière aqui apresentada, voltada para a avaliação dos conhecimentos de alunos de um curso de Licenciatura em Ciências Biológicas sobre evolução humana, configurou uma possibilidade real de aprendizagens múltiplas e possibilitar que os alunos percebam-se enquanto sujeitos de sua aprendizagem e protagonistas dos conteúdos de ensino. Além disso, como trata-se de futuros professores, a experiência possibilita a diversificação do repertório sobre instrumentos de avaliação e uma maneira de se refletir sobre as práticas docentes que são perpetuadas.

\section{Referências}

ALBUQUERQUE, Gregório; DIAS, Cynthia; SANTOS, João Paulo; MARINS, Hugo. O audiovisual como componente curricular da formação politécnica: a experiência da disciplina de Audiovisual da Escola Politécnica de Saúde Joaquim Venâncio. In: ALBUQUERQUE, Gregorio Galvão de; VELASQUES, Muza Clara Chaves; BATISTELLA, Renata Reis C. (Org.). Cultura, politecnia e imagem. Rio de Janeiro: EPSJV, 2017. p. 175-189.

BERTI, Andreza. Reverberações pedagógicas: As oficinas Minuto Lumière em uma escola catalã. Revista Tropos: Comunicação, Sociedade e Cultura. Rio Branco, v.9, n.1. Disponível em: https://periodicos.ufac.br/index.php/tropos/article/view/3431, acesso em 05/02/2021, jul. 2020.

BERGALA, Alain. A hipótese-cinema: Pequeno tratado de transmissão do cinema dentro e fora da escola. Rio de Janeiro: Booklink; CINEAD-LISE -FE/UFRJ, 2008.

CANI, Josiane; SANDRINI, Elizabete; SOARES, Gilvan; SCALZER, Kamila. Educação e COVD19: a arte de reinventar a escola mediando a aprendizagem "prioritariamente" pela TDIC. Revista IfesCiência, Vitória, v.6. Edição Especial, p. 23-39, 2020.

COMOLLI, Jean-Louis. La cámara. In: BALLÓ, Jordi y BERGALA, Alain. Motivos visuales del cine. Barcelona: Galaxia Gutenberg, S.L., pp: 116-121, 2016.

DE DEUS, Ana lara; OLIVEIRA, Valeska Maria. Cotidiano, cinema e formação: produções audiovisuais no curso de pedagogia. Ensaios Pedagógicos, Sorocaba, v.2, n.3, p. 63-68, 2018.

ELIAS, Carime; SILVEIRA, Paloma; COSTA, Janete; AXT, Margarete. Processos avaliativos em ambientes virtuais de formação: uma perspectiva interacional-dialógica. Estudos em Avaliação Educacional, São Paulo, v.26, n.61, p. 48-81, jan/ab. 2015.

FRESQUET, Adriana. Dossiê cinema e educação: uma relação sob a hipótese da alteridade. 
Revista Contemporânea de Educação, Rio de Janeiro, v.5, n.9, p. 1-7, jan.-jun. de 2010.

FUTUYMA, Douglas; KIRKPATRICK, Mark. Evolution. 4th Edition. Sunderland: Sinauer Assoicates, 2017.

GALEGO, Luís Gustavo; PEREIRA, Fernando. Planos, sequências e abstrações: a cinematografia e a educação. In: Formação de Professores: perspectivas teóricas e práticas na formação docente 2. Ponta Grossa: Atena Editora, 2020.

GUATTARI, Felix; ROLNIK, Suely. Micropolitica: cartografias do desejo. Petrópolis, RJ: Vozes, 2011.

LEVY, Pierre As Tecnologias da Inteligência: O futuro do pensamento na era da informática. São Paulo: Editora 34, 1993.

LINHARES, Ronaldo; LOUREIRO, Maria José; RAMOS, Fernando; ALCÂNTARA, Caio Mário. Avaliação das tecnologias digitais na docência: indicadores brasileiros e portugueses. Estudos em Avaliação Educacional, São Paulo, v. 28, n. 67, p. 12-31, jan./abr. 2017.

MCLUHAN, Marshall. Understanding Media: Os Meios de Comunicação como Extensões do Homem. São Paulo: Cultrix, 2004.

MEDRADO, Arthur; DINIZ, Margareth. O ensino da linguagem audiovisual e imagenssintomas. Revista Perspectivas Online: Humanas \& Sociais Aplicadas, Campos dos Goytacazes, v. 10, n. 28, p. 98-113, Jun. 2020.

RONDINI, Carina; PEDRO, Ketilin; DUARTE, Claúdia. Pandemia da COVID-19 e o ensino remoto emergencial: mudanças na prática pedagógica. Interfaces Científicas, Aracaju, v.10, n.1, p.41-57, 2020. 